\title{
Hydraulische Optimierung und Simulation von Qualitätsfragestellungen im Einzugsbereich von Horizontalfilterbrunnen
}

\author{
Sebastian Handl · Ernest Mayr · Reinhard Perfler
}

Online publiziert: 15. Juli 2019

(C) Der/die Autor(en) 2019

Zusammenfassung Horizontalfilterbrunnen haben in Bezug auf die Nutzung von Uferfiltrat vielfach Vorteile gegenüber anderen Brunnenarten. Aufgrund ihrer Lage zu nahegelegenen Oberflächengewässern werden bei Uferfiltratbrunnen jedoch oftmals besondere Schutzbestimmungen zur Kompensation von Schutzgebietseinschränkungen vorgesehen. Durch die Einstellung spezifischer hydraulischer Verhältnisse kann die Entnahme aus dem vorhandenen Grundwasserdargebot optimiert werden.

Zur Effizienzsteigerung bei den Förderraten werden die Strömungsverhältnisse mittels eines numerischen Modells untersucht, das die wesentlichen Einflussgrößen identifiziert und die Einhaltung der Entnahmebedingungen bei bestimmten Entnahmezuständen überprüft.

In dieser Arbeit werden zuerst die Grundlagen der numerischen Modellierung im Allgemeinen und der Umsetzung von horizontalen Filtersträngen im Besonderen dargestellt sowie die Erstellung eines 3D-Finite-DifferenzenModells für die Fallstudie beschrieben. Die Modellierung der Horizontalfilterbrunnen erfolgt nach dem Konzept der äquivalenten Durchlässigkeiten.

Das kalibrierte und validierte Modell wurde zur Berechnung von Bilanzen und Fließzeiten der zuströmenden Wassermengen bei 23 beobachteten stationären Systemzuständen angewendet. Fünf wesentliche Einflussgrößen

DI S. Handl (凶) • DI E. Mayr • PD DI Dr. R. Perfler

Department

Wasser-Atmosphäre-Umwelt, Institut für Siedlungswasserbau, Industriewasserwirtschaft und Gewässerschutz, Universität für Bodenkultur Wien,

Muthgasse 18, 1190 Wien, Österreich sebastian.handl@boku.ac.at auf die Strömungsverhältnisse wurden identifiziert.

\section{Schlüsselwörter}

Grundwassermodellierung . Horizontalfilterbrunnen - Äquivalente Durchlässigkeiten

\section{Hydraulic optimisation and} simulation of quality aspects in inflow areas of horizontal filter wells

Abstract Considering bank filtrate use, horizontal wells show numerous advantages compared to other well types. However, due to their proximity to nearby surface waters, bank filtration wells are often provided with special protection to compensate for protected area restrictions. By adjusting specific hydraulic conditions, the extraction from the existing groundwater supply can be optimized

In order to increase production rate efficiency, flow conditions are examined by means of a numerical model, which identifies key influencing parameters and verifies compliance of specified abstraction with extraction conditions.

The present article starts with the general basics of numerical modelling followed by its application for horizontal filter well in particular and the creation of a 3D finite difference model for the case study. The model used of the horizontal filter wells follows the concept of equivalent permeabilities.

The calibrated and validated model was used to calculate balances and flow times of inflowing water at $23 \mathrm{ob}-$ served steady state conditions. Five major influencing parameters for flow conditions were identified.

Keywords Groundwater modelling · Radial collector well · Equivalent conductivity

\section{Einleitung}

Horizontalfilterbrunnen haben in Bezug auf die Nutzung von Uferfiltrat vielfach Vorteile gegenüber anderen Brunnenarten. Aufgrund ihrer Lage zu nahegelegenen Oberflächengewässern werden bei Uferfiltratbrunnen jedoch oftmals besondere Schutzbestimmungen zur Kompensation von Schutzgebietseinschränkungen vorgesehen. Dazu können Grenzwerte für bestimmte oberflächengewässernahe Messpegel definiert werden, deren Einhaltung den Einzug von Wasser aus einem Oberflächengewässer ausschließen.

Um eine effiziente Ausschöpfung des Uferfiltratdargebots unter Einhaltung dieser Forderung zu entsprechen, müssen die Einflüsse der Rahmenbedingungen (z.B. Wasserstände der Oberflächengewässer) auf die Strömungsverhältnisse untersucht werden.

Eine exakte analytische Bemessung von Horizontalfilterbrunnen ist aufgrund der komplexen Strömungsverhältnisse in der Praxis selten möglich (Mutschmann und Stimmelmayr 2011). Zum Beispiel gehen die empirisch gewonnenen Bemessungstafeln nach Falcke (1952, zit. in Mutschmann und Stimmelmayr 2011) von einer symmetrischen Aufteilung der Filterstränge in alle Richtungen aus.

Zum einen ist die Aufteilung der Filterstränge in den meisten Fällen nicht symmetrisch, da oftmals andere Gesichtspunkte bei der Anpassung an natürliche Gegebenheiten ausschlaggebend sind. Zum anderen ergeben sich durch die spezielle Lage von Brunnenstandorten an und zwischen einzelnen Oberflächengewässern (z. B. Auengebiet, Altarmanbindung etc.) komplexe, im Jahresverlauf stark variierende Randbedingungen für die Berechnung der Strömungsverhältnisse. Gerade diese Umstände machen die Anwendung von numerischen Modellen bei der Bear- 
beitung entsprechender Fragestellung besonders interessant.

\section{Aufgabenstellung und Zielsetzung}

In der gängigen Praxis der Modellierung von Horizontalfilterbrunnen werden die Fließverhältnisse im Brunnen bzw. in den Filtersträngen nicht berücksichtigt. Neuere Methoden bieten die Möglichkeit, die Wechselwirkung zwischen Brunnen und Grundwasserleiter in die Berechnung der Strömungsverhältnisse einfließen zu lassen. Dadurch kann der Vereinfachungsgrad von Modellen reduziert werden und so die Wirklichkeit realitätsnäher abgebildet werden. Im Folgenden ist ein Überblick über die verschiedenen möglichen Konzepte dargestellt. Im Rahmen eines Fallbeispiels wird eines der vorgestellten Konzepte zur simultanen Lösung der Brunnen- und Grundwasserhydraulik angewendet und seine schrittweise Umsetzung beschrieben.

Übergeordnet wurde dazu ein Projekt zur hydraulischen Optimierung und Simulation von Qualitätsfragestellungen im Einzugsbereich von Horizontalfilterbrunnen durchgeführt, auf das hier zeitweise Bezug genommen wird. Zur Erstellung der Datenbasis für die Kalibrierung der Materialparameter des beschriebenen Grundwasserleiters wurde ein Pumpversuch durchgeführt. Dieser erstreckt sich über etwa zwei Wochen und besteht aus einer Aufspiegelungs- und einer Absenkphase. Die Validierung des Modells wurde anhand von Pumpversuchen aus den Jahren 2009 und 2010 durchgeführt. Die Wassertemperatur im Untersuchungsgebiet unterliegt relativ hohen jahreszeitlichen Schwankungen. Dem Einfluss der Temperatur auf die Viskosität des Grundwassers kann im Modell nur durch eine Adaptierung der Durchlässigkeitsbeiwerte Rechnung getragen werden. Im Zuge der Validierung soll die Notwendigkeit der Berücksichtigung dieses Effekts durch Anwendung bekannter Formeln gezeigt werden. Unter Verwendung des Softwaremoduls PMPATH (integriert in PMWIN - Chiang und Kinzelbach 2001) wurden im Projekt, mittels Modellierung des advektiven Stofftransportes, die Fließzeiten des Wassers bzw. Laufzeiten möglicher Verunreinigungen von den Modellrändern zu den Brunnen berechnet. Das kalibrierte und validierte Strömungsmodell dient im Folgenden als Instrument, um Bilan- zen der zuströmenden Wassermengen aus den nahegelegenen Oberflächengewässern bei unterschiedlichen Rahmenbedingungen und Förderraten $\mathrm{zu}$ generieren. Aus umfangreichen Daten mehrerer Jahre werden stationäre Systemzustände mit verschieden Fördermengen ausgewählt, um die Bandbreite der auftretenden Einflussgrößen und Randbedingungen auf die Modellaussagen, Zuflussbilanz und Fließzeiten repräsentativ darstellen zu können.

In Abhängigkeit von den die Rahmenbedingungen definierenden Parametern soll eine Optimierung der Fördermenge dahingehend durchgeführt werden, dass eine Mindestaufenthaltszeit des dem Grundwasserleiter zuströmenden Wassers aus den Oberflächengewässern von 60 Tagen eingehalten wird.

\section{Grundlagen und Methodik}

In dieser Arbeit werden die Beschreibung der Hydraulik und Modellierung von Horizontalfiltersträngen in den Vordergrund gerückt, weshalb auf die allgemeinen Grundlagen von Strömungsund Transportprozessen im Grundwasser nicht weiter eingegangen wird.

\subsection{Hydraulik der Horizontalfilterstränge}

\subsection{Physikalische Beschreibung}

Nemeček (2006) beschreibt die Hydraulik von Filtersträngen physikalisch. Die Wasserentnahme im Brunnenschacht eines Horizontalfilterbrunnens bewirkt dort eine Absenkung $h_{s}$ gegenüber dem Ruhewasserspiegel. Das entstehende Potenzialgefälle drückt die Energieverluste am Fließweg in Meter Wassersäule aus. Diese setzen sich aus den Verlusten bei der Durchströmung des Aquifers $\left(h_{f}\right)$, den Verlusten beim Eintritt in das Filterrohr $\left(h_{e}\right)$ und den Rohrreibungsverlusten beim Durchströmen des Filterrohres $\left(h_{r}\right)$ zusammen (Gl. 1, Abb. 1).

$$
h_{s}=h_{f}+h_{e}+h_{r}
$$

Die hydraulischen Eigenschaften des Filterstrangs finden im Eintrittswiderstand und den Rohrreibungsverlusten Ausdruck. Nemeček (2006), wie auch Chen et al. (2003), stellten fest, dass aufgrund der geringen Eintrittsgeschwindigkeit die Verlusthöhe durch das Passieren der Schlitze im Filterrohrmantel gegenüber den Rohrreibungsverlusten vernachlässigbar gering ist.

Olson und Wright (1990, zit. bei Chen et al. 2003) geben eine Übersicht $\mathrm{zu}$ Verlusten in Rohrleitungen $\left(h_{v}\right)$. Das Strömungsregime in Druckrohrleitungen kann in Abhängigkeit von der Reynolds-Zahl (Re), dem Quotienten aus Rohrinnendurchmesser (d) und der Rauigkeit der Rohrinnenwand $(\mathrm{k})$ in drei Hauptbereiche und zwei Übergangsbereiche gegliedert werden (Abb. 2).

1. Bei laminarer Strömung ist der Rohrreibungsverlust linear von der mittleren Strömungsgeschwindigkeit abhängig $\left(h_{v} \sim v\right)$,

2. im glatten turbulenten Bereich ist $h_{v}$ proportional zu v $\mathrm{v}^{1,75}$ und

3. im rauen turbulenten Bereich proportional zum Quadrat der Fließgeschwindigkeit $\left(\mathrm{h}_{\mathrm{v}} \sim \mathrm{v}^{2}\right)$.

Mohamed und Rushton (2006) stellen bei ihrer Untersuchung von horizontalen Filtersträngen in einem flachgründigen Grundwasserleiter fest, dass auch bei geringeren Fließgeschwindigkeiten in Filterrohren das Auftreten von turbulenten Strömungsverhältnissen durch den Einfluss der radialen Zuströmung wahrscheinlich ist.

In der Literatur finden sich folgende Angaben zu den Rauigkeiten der Rohrinnenwand bei Filterrohren: Stack (1958, zit. bei Grombach et al. 2000) gibt ein Verhältnis zwischen Voll- zu Filterrohr von 0,25 an. Nemeček (2006) ermittelt durch Laborversuche (bandagierte Ranney-Rohre; DN200; Q=301/s) eine Verlusthöhe von $0,2 \mathrm{~m}$ bei $30 \mathrm{~m}$ Stranglänge. Dieser Wert entspricht einer Wandrauigkeit von $0,83 \mathrm{~mm}$.

\subsubsection{Numerische Modellierung horizontaler Filterstränge}

In der numerischen Modellierung horizontaler Filterstränge in porösen Grundwasserkörpern werden drei verschiedene Konzepte angewendet: Filterstrang als Randbedingung Der Filterstrang wird als lineare Senke dargestellt. Auf diese kann entweder die Randbedingung erster Art oder die Randbedingung zweiter Art angewandt werden. Dabei wird die Standrohrspiegelhöhe bzw. die Entnahme entlang der Senke konstant gehalten. Dieses Konzept findet unter anderem bei Kawecki (2000) und Sun und Zhan (2005) Anwendung. 

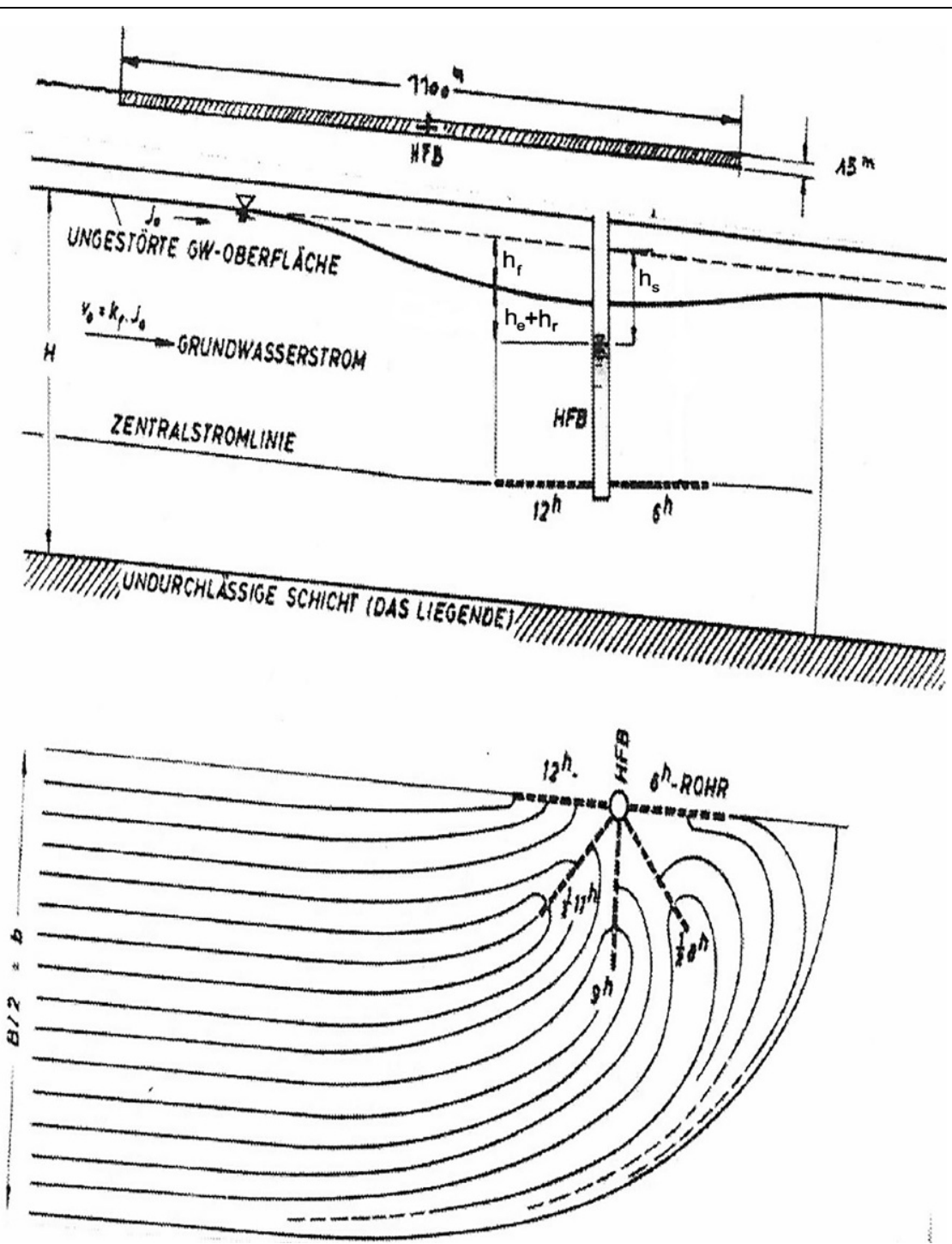

Abb. 1 Ausbildung des Absenktellers in einem geneigten Aquifer (Nemeček 2006)

Sowohl Tarshish (1992) als auch Nemeček (2006) stellen fest, dass die Verteilung der Entnahme und in weiterer Folge auch jene der Potenzialhöhe entlang eines Filterstrangs nicht konstant sind. Abb. 3 zeigt die Zuströmung zu einem Horizontalfilterstrang (New Solution). Die Beaufschlagung in der Filterrohrspitze $(\mathrm{x}=0)$ ist am höchsten.

Zhan und Zlotnik (2002) untersuchen den Einfluss durch die vereinfachte Annahme von konstanten Entnahmemengen (Conventional Solution in Abb. 3) bzw. Standrohrspiegelhöhen entlang des Filterstrangs. Sie stellen fest, dass der begangene Fehler in einer Distanz von 5-fachem Rohrdurchmesser $10 \%$ beträgt und mit zunehmender Entfernung vom Filterstrang schnell auf ein Niveau von weniger als einem Prozent abnimmt.
Aquifer und kann für den jeweiligen Strömungszustand im Rohr mit den Gln. 2, 3 und 4 berechnet werden.

$$
\begin{aligned}
& k_{f, \text { equ }}=\left\{\begin{array}{c}
\frac{g d}{32 v_{\text {kin }}} \\
R e<2300 \\
\frac{2 g d}{0,316} \cdot\left(\frac{d}{v_{\text {kin }}}\right)^{0,25} \cdot \frac{1}{\nu^{0,25}} \\
3000<R e<100.000 \\
8 g d \cdot\left(\log _{10} 3,71 \frac{d}{k}\right)^{2} \cdot \frac{1}{v} \\
R e>100.000
\end{array}\right. \\
& v=\frac{v_{\mathrm{rohr}} \cdot A_{\mathrm{rep}}}{A_{\mathrm{rohr}}} \\
& R e=\frac{v_{\text {rohr }} \cdot d}{v_{\text {kin }}}
\end{aligned}
$$

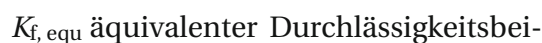
wert $[\mathrm{m} / \mathrm{s}]$

$g$ Erdbeschleunigung 9,81 [m/s $\left./ \mathrm{s}^{2}\right]$

$d \quad$ Innenrohrdurchmesser [m]

$v_{\text {kin }} \quad$ kinematische Viskosität $\left[\mathrm{m}^{2} / \mathrm{s}\right]$

$k$ Innenwandrauigkeit [m]

Re Reynoldszahl in Rohrleitungen [ ]

$v_{\text {rohr }}$ Fließgeschwindigkeit im Rohr $[\mathrm{m} / \mathrm{s}]$

$A_{\text {rep }}$ Querschnittsfläche der Zelle die das Rohr repräsentiert $\left[\mathrm{m}^{2}\right]$

$A_{\text {rohr }}$ Querschnittsfläche der Rohres $\left[\mathrm{m}^{2}\right]$

Es ist eine sehr detaillierte räumliche Diskretisierung der Filterstränge mit Zellengrößen kleiner gleich $1 \mathrm{~m}$ für die Umsetzung dieses Konzeptes in einem Modell erforderlich. Dieser Umstand stellt laut Kelson (2012) einen wesentlichen Nachteil dieser Methodik dar.

Kopplung von Rohr- und Grundwasserströmungsmodell Tarshish (1992) stellt ein kombiniertes mathematisches Modell für stationäre Bedingungen vor. Es berücksichtigt turbulente Fließzustände im Filterrohr. Als Schnittstelle wirkt eine lineare Senke mit variablen Entnahmemengen entlang des Strangs. Eine vergleichbare numerische Umsetzung, die durch Aufteilung der Brunnenentnahme auf mehrere Knoten in einem Finite-Differenzen-Modell realisiert wird, findet sich bei Kelson (2012). Eine Anpassung der räumlichen Diskretisierung ist bei dieser Methodik nicht notwendig, wodurch die Anwendbarkeit auf Modelle in regionalem Maßstab gegeben ist.

Chen et al. (2003) wenden das Konzept der äquivalenten Durchlässigkeiten auf ein instationäres Modell an und überprüfen das numerischen Modell mittels eines Laborversuchs. Da bei einem instationären Modell zu unterschiedlichen Zeitpunkten verschiede- 


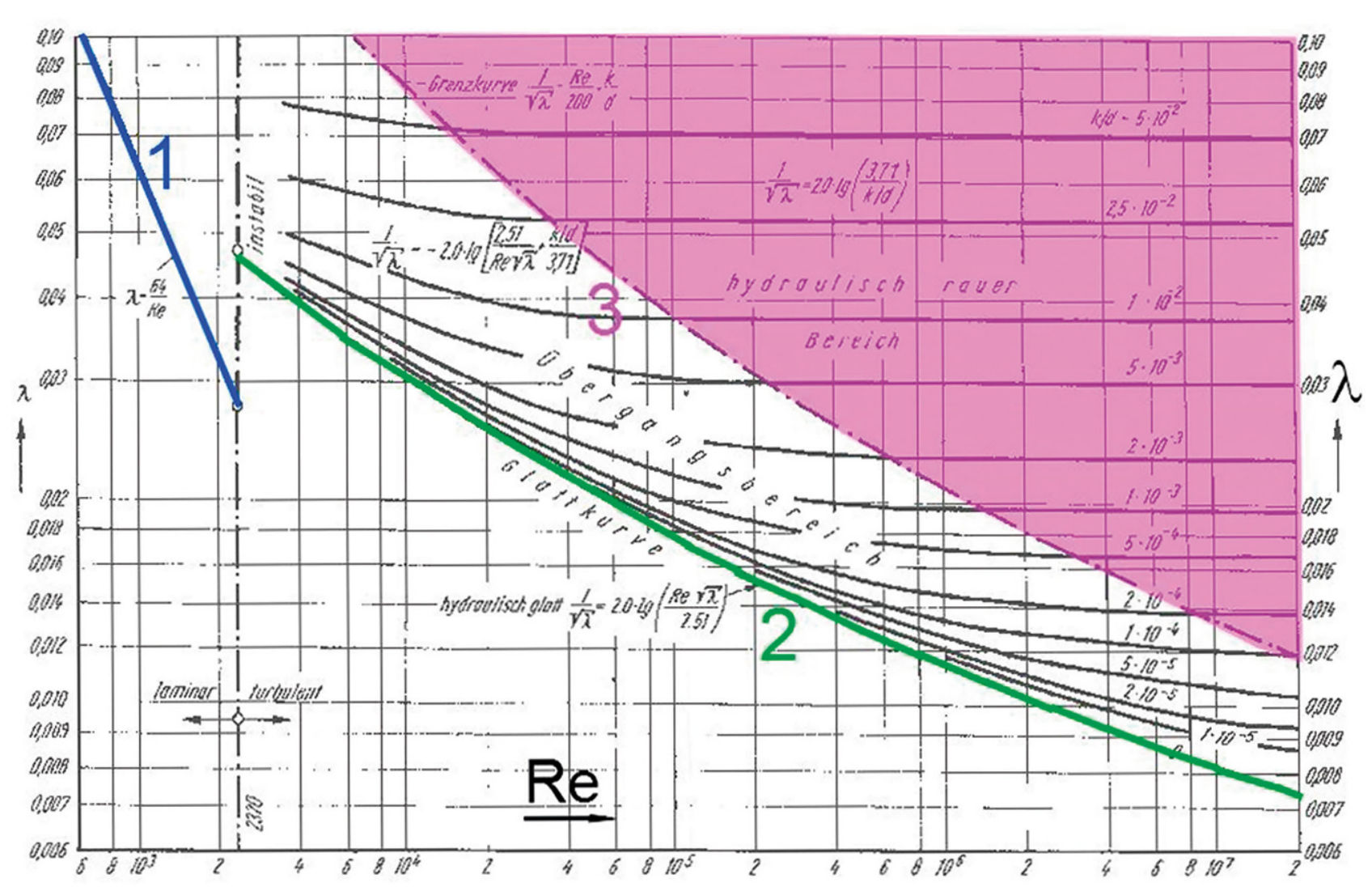

Abb. 2 Hauptbereiche des Strömungsregimes im Moody-Diagramm (Bollrich und Preißler 2000)

ne Strömungsverhältnisse im Filterrohr vorherrschen können, müssen die äquivalenten Durchlässigkeitsbeiwerte der Filterstränge iterativ für jeden Zeitschritt von einem zusätzlichen Modell, welches mit dem Grundwassermodell gekoppelt wird, bestimmt werden.

Mohamed und Rushton (2006) entwickeln ein Konzept zur Kopplung von drei Modellen, welche den regionalen Grundwasserfluss, das Zuströmen vom Aquifer zum horizontalen Filterstrang und die Strömungsverhältnisse im Filterrohr selbst repräsentieren. Die Interaktion zwischen Aquifer und Brunnen wird mittels einer linearen Beziehung zwischen Zufluss und Potenzialunterschied innerhalb und außerhalb des Filterstranges nachgebildet. Der Zuflussfaktor oder -koeffizient muss dabei empirisch ermittelt werden. Es werden somit zwar einerseits die Verluste beim Durchströmen der Filterschlitze berücksichtigt, dies geschieht andererseits jedoch stark abstrahiert.

\subsection{Aufbau des 3D-Strömungsmodells}

Als Modellierungssoftware wurde Processing Modflow verwendet. MOD-
FLOW (Harbaugh 2005) ist ein 3D-Finite-Differenzen-Modell, das vom U.S. Geological Survey entwickelt wurde. PMWIN (Processing Modflow für Windows) integriert MODFLOW in eine grafische Benutzeroberfläche und bietet eine Vielzahl an Modulen zur Modellierung von Grundwasserströmung und Stofftransport in der gesättigten Bodenzone.

Nach der Wahl der Modellierungssoftware gliedert sich der Aufbau des dreidimensionalen Strömungsmodells in vier Bereiche:

- Erstellung des Modellkonzepts und Eingabe der Geometrie:

Dazu zählt die Definition der Modellgrenzen in der Horizontalen (Lage von Randzellen und Wahl der Randbedingungsart) und Vertikalen (Festlegung des Grundwasserstauers). Auf die Umsetzung des vorgestellten Konzepts der äquivalenten Durchlässigkeiten zur Modellierung der Brunnenschächte und Horizontalfilterstränge folgt die räumliche Diskretisierung und die Definition von Beobachtungspunkt im Modell, um Vergleiche der Simulation mit
Messwerten der Grundwasserpegel anstellen zu können.

- Kalibrierung des Modellparameters: Nach der Durchführung eines Pumpversuchs zur Datenerhebung können Bereiche mit homogenen geohydraulischen Parametern im Modell definiert werden. Des Weiteren werden aus der Messung die Werte zur Eingabe von Anfangs- und Randbedingungen ermittelt und die zeitliche Diskretisierung des Simulationszeitraumes durchgeführt. Bei der eigentlichen Kalibrierung werden nun die geohydraulischen Parameter variiert, um eine möglichst gute Übereinstimmung der modellierten Wasserstände mit den gemessenen zu erreichen.

- Validierung:

Dafür werden Datensätze ausgewählt, welche als unabhängig vom Kalibrierungsdatensatz betrachtet werden können. Aus ihnen werden wiederum die Werte für Anfangsund Randbedingungen erstellt und der Simulationszeitraum wird diskretisiert. Im Zuge der Überprüfung der gewählten Parameterkombination wird gleichzeitig auch der Einfluss 


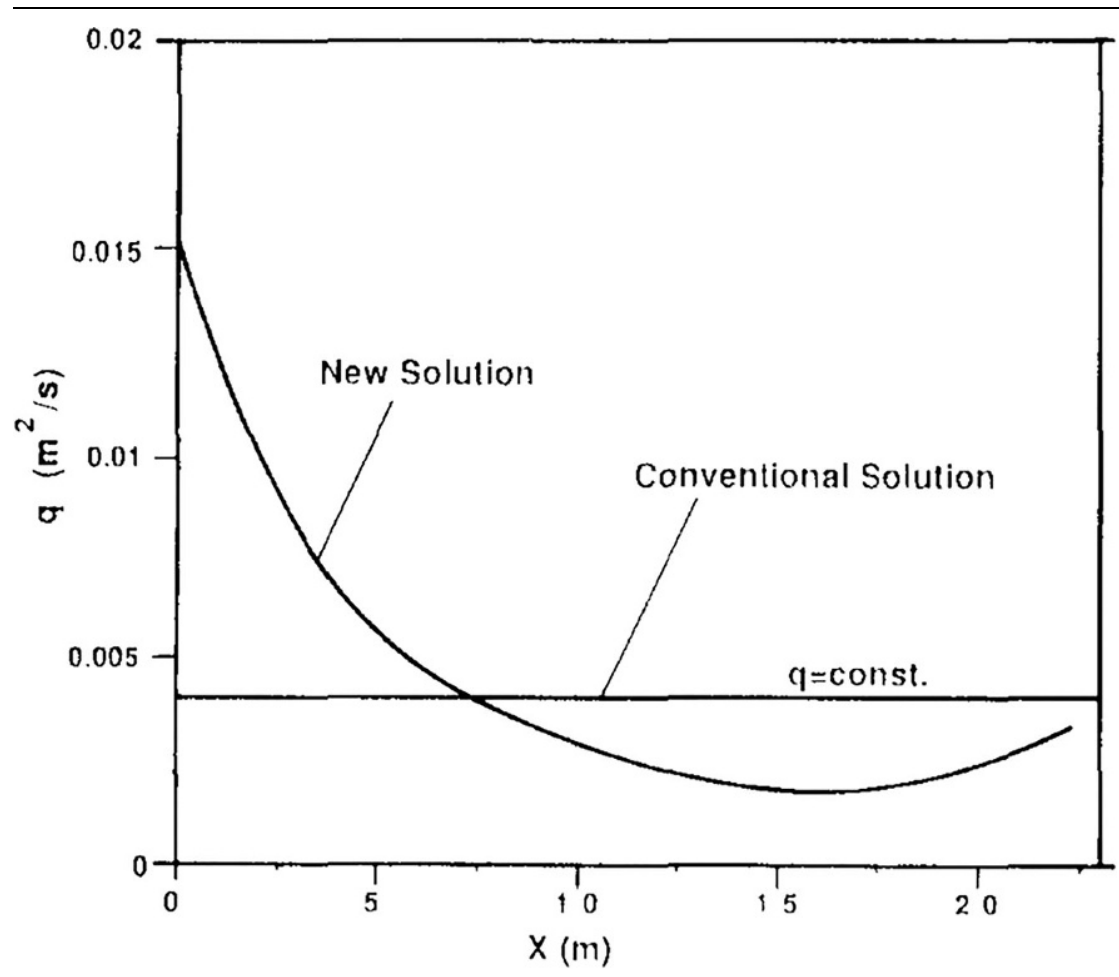

Abb. 3 Zuströmung entlang eines Horizontalfilterstrangs (Tarshish 1992)

der Temperatur auf die Durchlässigkeitsbeiwerte überprüft.

\subsection{Abstrahierung von Horizontalfilterbrunnen}

Die Modellierung der Horizontalfilterbrunnen erfolgte im Anwendungsbeispiel nach dem Konzept der äquivalenten Durchlässigkeiten. Dabei wird $k_{\text {f,equi }}$ im instationären Modell nicht für alle Strömungsregime angepasst, sondern es werden lediglich turbulente Verhältnisse berücksichtigt. Es werden äquivalente Durchlässigkeitsbeiwerte für relevante Entnahmemengen ermittelt. Daraufhin werden $k_{\text {f,equi-Werte }}$ gewählt, die für die Verlusthöhen vernachlässigbar geringe Abweichungen bei relevanten Durchflüssen ergeben. Der Berechnungsgang ist im Folgenden konzeptuell dargestellt. Die Ermittlung des Rohrreibungsgefälles erfolgt nach Bollrich und Preißler (2000). Die geometrischen Eingangswerte des Brunnens ( $\left.n_{\text {Stränge, }} d_{\text {Rohr }}, l_{\text {Filterrohr }}\right)$ und die Abmessungen der Zelle, durch die der Filterstrang repräsentiert wird ( $\left.A_{\text {Modell }}\right)$, sind zu ermitteln. Für die dynamische Viskosität $\left(v_{\text {kin }}\right)$ wird für diese Berechnung der Wert $1,31 * 10^{-6} \mathrm{~m}^{2} / \mathrm{s}$ (bei $10^{\circ} \mathrm{C}$ ) verwendet. Die Rohrwandrauigkeit $(k)$ wird entsprechend der Emp-

$$
\begin{aligned}
& h_{v, \text { Modell,gew. }}=I_{v, \text { Modell }} \cdot l_{\text {Filterrohr }} \\
& h_{v, \text { Rohr }}=\lambda \cdot \frac{l}{d} \cdot \frac{v^{2}}{2 \cdot g}
\end{aligned}
$$

\subsection{Modellierung des Stofftransports mittels dispersionsfreier Näherung}

Zur Ermittlung der Fließwege möglicher Verunreinigungen bzw. der Aufenthaltszeit des Grundwassers vom Modellrand $\mathrm{zu}$ den Brunnen wurde die Software PMPATH (Version 6.1.2) verwendet. Das Programm berechnet den advektiven Stofftransport im Grundwasser. Es verwendet die Simulationsergebnisse aus Processing Modflow und einen semianalytischen Ansatz zur Verfolgung von Partikeln.

Unter Anwendung des Gesetzes von Darcy werden aus dem Standrohrspiegelhöhenfeld die Volumenströme durch jede Zellenaußenfläche berechnet. Daraus wird die Abstandsgeschwindigkeit bzw. die mittlere Geschwindigkeit in den Poren des Grundwasserleiters durch jede Zellenaußenfläche berechnet.

Mit der Annahme, dass sich die Geschwindigkeitskomponenten innerhalb jeder Zelle nur linear ändern, kann für jeden Punkt im Modell der Hauptvektor der Grundwasserströmung berechnet werden. Für vorgegebene Partikelstartpunkte können so die Fließwege bzw. der Aufenthaltsort des Partikels zu einem späteren Zeitpunkt berechnet werden.

Um die Laufzeiten möglicher Schadstoffe vom Modellrand zum Brunnen zu ermitteln, wurde in der Mitte der Außenfläche jeder Modellrandzelle im Layer 2 ein Partikel positioniert. Durch Simulation der Fließwege unter kontinuierlicher Steigerung der Simulationszeit konnte so der Zeitpunkt des Eintretens des ersten Partikels mit Ursprung am jeweiligen Modellrand in eine den Brunnen repräsentierende Zelle ermittelt werden.

$$
\begin{aligned}
& A_{\text {Rohr }}=\frac{d_{\text {Filterrohr }}^{2} \cdot \pi}{4} \\
& v_{\text {Rohr }}=\frac{Q_{\text {Brunnen }}}{n_{\text {Stränge }} \cdot A_{\text {Rohr }}} \\
& R_{\text {Rohr }}=\frac{v_{\text {Rohr }} \cdot d_{\text {Rohr }}}{v_{\text {kin }}} \\
& \lambda_{\text {rau }}=\frac{1}{4 \cdot \log ^{2}\left(\frac{3,71 \cdot d_{\text {Rohr }}}{k}\right)} \\
& I_{\nu, \text { Rohr }}=\frac{\lambda_{\text {rau }} \cdot v^{2}}{2 \cdot g \cdot d_{\text {Rohr }}} \cdot A_{\text {Rohr }} \\
& k_{f, \text { equi,calc }}=\frac{\nu_{\text {Rohr }}}{A_{\text {Modell }} \cdot I_{\nu, \text { Rohr }}} \\
& v_{\text {Modell }}=\frac{Q_{\text {Brunnen }}}{n_{\text {Stränge }} \cdot A_{\text {Modell }}} \\
& I_{\text {Modell }}=\frac{v_{\text {Modell }}}{k_{f, \text { equi,gew }}}
\end{aligned}
$$

\subsection{Szenarienentwicklung zur Quantifizierung der Modellgenauigkeit}

Die Quantifizierung der Modellgenauigkeiten bzw. die Ermittlung des Schwankungsbereichs der jeweiligen Ergebnisse erfolgte unter Anwendung der Szenarientechnik.

Durch Erhöhung bzw. Verringerung der Durchlässigkeitsbeiwerte um $10 \%$ werden mit der Ursprungsvariante drei 
Tab. 1 Güte der Szenarien (Verschlechterung in Bezug auf das Grundszenario) der stationären Modellierung des Zustandes am 26.04.2012

\begin{tabular}{l|lll|}
\hline Szenario & & $\begin{array}{l}\text { MQA } \\
{[\mathrm{cm}]}\end{array}$ & \\
\hline$K_{\mathrm{f}}$ & $-10 \%$ & 13,77 & $(33 \%)$ \\
\hline$K_{\mathrm{f}}$ & $+0 \%$ & 10,38 & $(0 \%)$ \\
\hline$K_{\mathrm{f}}$ & $+10 \%$ & 12,97 & $(25 \%)$ \\
\hline
\end{tabular}

in sich plausible Szenarien entwickelt. Die Güte der verschiedenen Szenarien ist anhand der Untersuchungen der Feinkalibrierung am stationären Modell ersichtlich und in Tab. 1 dargestellt.

Das Grundszenario gilt aufgrund der geringsten Abweichungen zur Messung als wahrscheinlichste Abbildung der Wirklichkeit. Als Schwankungsbereich der Ergebnisse wird die Differenz aus Ursprungsszenario und jenem Szenario angegeben, welches das jeweilige Modellergebnis negativ beeinflusst.

Durch die Verringerung der Durchlässigkeitsbeiwerte verschiebt sich die Bilanz der Zuflüsse ins Modellgebiet, wodurch mehr Zufluss aus Richtung eines Oberflächengewässers erfolgt. Der Schwankungsbereich bezüglich dieser Modellaussage wird daher aus der Differenz der Ergebnisse von Ursprungsszenario und Szenario $\left(k_{\mathrm{f}}-10 \%\right)$ angegeben.

Ähnlich verhält es sich mit der Fragestellung um die Laufzeit vom Oberflächengewässer zum Brunnen. Um eine eher konservative Aussage zu treffen, wird der Schwankungsbereich zwischen Ursprungsszenario und dem Szenario mit verringerten Durchlässigkeitsbeiwerten angegeben.

Die Laufzeit des Wassers aus dem Oberflächengewässer zu den Brunnen ist im Szenario mit höheren Durchlässigkeitsbeiwerten geringer. Die Schwankungsbreite wird daher zwischen den Ergebnissen des Ursprungsszenarios und dem Szenario mit erhöhten Durchlässigkeitsbeiwerten $\left(k_{\mathrm{f}}+10 \%\right)$ angegeben.

\section{Ergebnisse}

\subsection{Ergebnisse von Kalibrierung und Validierung}

Die Berücksichtigung des Einflusses der Temperatur auf das Strömungsverhalten durch Adaptierung der Durchlässigkeitsbeiwerte mittels eines globalen Temperaturfaktors zeigt für beide Validierungsdatensätze und in Bezug auf beide Gütekriterien eine deutliche Erhöhung der Modellgüte.

\subsection{Anwendung und Interpretation der Modellierungsergebnisse}

Mit dem kalibrierten Strömungsmodell ist es möglich, für beobachtete Zustände Bilanzen der zuströmenden Wassermengen aus Richtung der Oberflächengewässer zu erstellen. Die Auswertung modellierter Wasserstände mithilfe des Softwaremodules PMPATH zur dispersionsfreien Näherung des Stofftransports ermöglicht die Ermittlung von Fließzeiten im Modell.

Durch globale Variation der Durchlässigkeitsbeiwerte wurden für jedes Modellierungsergebnis ungünstige Szenarien entwickelt, um die Modellgenauigkeit bzw. die Schwankungsbreiten der Modellierungsergebnisse darzustellen.

Bestimmte Einflussgrößen definieren das vorhandene Uferfiltratdargebot, das durch die Förderraten in den Brunnenfeldern ausgeschöpft werden kann. Übersteigt die Entnahme das vorhandene Dargebot, kommt es zu einem verstärkten Zufluss von Wasser aus Richtung von Oberflächengewässern.

Zur Analyse des Einflusses der Rahmenbedingungen auf die Strömungsverhältnisse wurden 23 beobachtete stationäre Systemzustände (und ein
Nullzustand) mit dem Modell nachgebildet und Strömungsbilanzen sowie Fließzeiten von den Modellrändern zu den Brunnen ermittelt.

Bei der Analyse der Ergebnisse der numerischen Modellierung wurden zwei wesentliche Erkenntnisse gewonnen:

1. Der Zufluss aus Richtung des Oberflächengewässers steht in engem $\mathrm{Zu}$ sammenhang mit dem Verhältnis der Potenzialdifferenzen im Bereich des betrachteten Horizontalfilterbrunnens.

2. Die kürzeste Fließzeit vom Modellrand aus Richtung des Oberflächengewässers zum Brunnen steht in engem Zusammenhang mit der Gesamtzuflussrate aus Richtung des Oberflächengewässers.

Durch Kombination dieser Erkenntnisse ist es möglich, einen betrieblichen Fließzeitparameter zu definieren. Die Förderrate im Brunnen soll anhand einer definierten Mindestaufenthaltszeit des zuströmenden Wassers aus Richtung des Oberflächengewässer gesteuert werden. Hierbei kann z.B. die Definition der Mindestaufenthaltszeit an den Vorgaben der ÖVGW-Richtlinie W72 „Schutz- und Schongebiete“ für die Grenzdefinition der Schutzzone II (entspricht 60 Tage) herangezogen werden (ÖVGW 2004).

Funding Open access funding provided by University of Natural Resources and Life Sciences Vienna (BOKU).

Open Access Dieser Artikel wird unter der Creative Commons Namensnennung 4.0 International Lizenz (http:// creativecommons.org/licenses/by/4. $0 /$ deed.de) veröffentlicht, welche die Nutzung, Vervielfältigung, Bearbeitung, Verbreitung und Wiedergabe in jeglichem Medium und Format erlaubt, sofern Sie den/die ursprünglichen $\mathrm{Au}$ tor(en) und die Quelle ordnungsgemäß nennen, einen Link zur Creative Commons Lizenz beifügen und angeben, $o b$ Änderungen vorgenommen wurden. 
Bollrich, G., Preißler, G. (2000): Technische Hydromechanik Band 1 - Grundlagen, 5. Aufl., Verlag für Bauwesen, Berlin

Chen, C., Wan, J., Zhan, H. (2003): Theoretical and experimental studies of coupled seepagepipe flow to a horizontal well, Journal of Hydrology, vol. 281, 1-2, p. 159-171

Chiang, W. H. and Kinzelbach, W. (2001): 3DGroundwater Modeling with PMWIN. First Edition. Springer, Berlin Heidelberg New York. ISBN 3-540 67744-5, 346 pp.

Grombach, P., Merkl, G, Haberer, K., Trüieb, E U. (2000): Handbuch der Wasserversorgungstechnik, 3. Aufl., Oldenbourg Industrieverlag, München-Wien

Harbaugh, A. W. (2005): MODFLOW-2005, The US Geological Survey modular groundwater model: The groundwater flow process. US Department of the Interior; USGS Numbered Series 6A16. https://doi.org/10.3133/tm6A16
Kawecki, M. W. (2000): Transient Flow to a Horizontal Water Well, Ground Water, vol. 38, 6 , p. $842-850$

Kelson, V. (2012): Predicting Collector Well Yields with MODFLOW, Ground Water, vol. 50, 6, p. 918-926

Mohamed, A., Rushton, K. (2006): Horizontal wells in shallow aquifers: Field experiment and numerical model, Journal of Hydrology, vol. 329 1-2, p. 98-109

Mutschmann, J., Stimmelmayr, F. (2011): Taschenbuch der Wasserversorgung, 15. Aufl., Vieweg+Teubner Verlag, Wiesbaden

Nemeček, E. P. (2006): Horizontalfilterbrunnen, Schriftenreihe zur Wasserwirtschaft TU Graz, Band 45, Eigenverlag des Instituts für Siedlungswasserwirtschaft und Landschaftswasserbau der TU Graz

ÖVGW (2004): Richtlinie W72 - Schutz- und Schongebiete, Österreichische Vereinigung für das Gas- und Wasserfach
Sun, D., Zhan, H. (2005): Flow to a horizontal well in an aquitard-aquifer system, Journal of Hydrology, vol. 321, 1-4, p. 364-376

Tarshish, M. (1992): Combined Mathematical Model of Flow in an Aquifer-Horizontal Well System, Ground Water, vol. 30, 6, p. 931-935 Wang, W., Zhang, G. (2007): Numerical simulation of groundwater flowing to horizontal seepage wells under a river, Hydrogeology Journal, vol. 15, 6, p. 1211-1220

Zhan, H., Zlotnik, V. A. (2002): Ground water flow to horizontal and slanted wells in water table aquifers, Water Resources Research, vol. 38,7, p.13-1-13-11

Hinweis des Verlags Der Verlag bleibt in Hinblick auf geografische Zuordnungen und Gebietsbezeichnungen in veröffentlichten Karten und Institutsadressen neutral. 